\title{
Prelacteal feeding practice and its associated factors among mothers having children less than 2 years of age in East Africa: a multilevel analysis of the recent demographic and health surveys
}

\author{
Achamyeleh Birhanu Teshale ${ }^{1 *}$ (D), Misganaw Gebrie Worku², Zemenu Tadesse Tessema ${ }^{1}$ and \\ Getayeneh Antehunegn Tesema ${ }^{1}$
}

\begin{abstract}
Background: Prelacteal feeding is a major public health problem that increases the risk of morbidity and mortality in children. It also result delayed breastfeeding initiation and interferes with exclusive breastfeeding. Although numerous studies have been done on prelacteal feeding in individual East African countries, most of them did not consider community-level factors that could affect the likelihood of prelacteal feeding. This study, thus, aimed to assess the pooled prevalence and associated factors of prelacteal feeding practice in East Africa.

Methods: We used pooled data from the 12 east Africa countries Demographic and Health Surveys (DHS). A total weighted sample of 33,423 women was included in the final analysis. We employed multilevel logistic regression analysis to assess factors associated with prelacteal feeding practice. Finally, the Adjusted odds ratio (AOR) with 95\% Confidence $(\mathrm{Cl})$ interval was reported and variables with $p$ value $<0.05$, in the multivariable analysis, were declared to be significant predictors of prelacteal feeding practice.

Result: In this study, the pooled prevalence of prelacteal feeding practice was $11.85 \%$ (95\%Cl: 11.50, 12.20) with great variation between countries, ranging from 3.08\% (95\%Cl: 2.35, 3.81) in Malawi to 39.21\% (95\%Cl: 36.36, 42.06) in Comoros. Both individual and community-level factors were associated with prelacteal feeding practice. Of the individual-level factors, home delivery, multiple birth, cesarean delivery, non-exposure to media, delayed initiation of breastfeeding, and being a small-sized baby were associated with higher odds of prelacteal feeding practice. Among the community-level factors, rural residence and higher community-level of media exposure were associated with lower odds of prelacteal feeding practice.
\end{abstract}

\footnotetext{
* Correspondence: achambir08@gmail.com

${ }^{1}$ Department of Epidemiology and Biostatistics, Institute of Public Health, College of Medicine and Health Sciences, University of Gondar, Gondar, Ethiopia

Full list of author information is available at the end of the article
}

(c) The Author(s). 2021 Open Access This article is licensed under a Creative Commons Attribution 4.0 International License, which permits use, sharing, adaptation, distribution and reproduction in any medium or format, as long as you give appropriate credit to the original author(s) and the source, provide a link to the Creative Commons licence, and indicate if changes were made. The images or other third party material in this article are included in the article's Creative Commons licence, unless indicated otherwise in a credit line to the material. If material is not included in the article's Creative Commons licence and your intended use is not permitted by statutory regulation or exceeds the permitted use, you will need to obtain permission directly from the copyright holder. To view a copy of this licence, visit http://creativecommons.org/licenses/by/4.0/. The Creative Commons Public Domain Dedication waiver (http://creativecommons.org/publicdomain/zero/1.0/) applies to the data made available in this article, unless otherwise stated in a credit line to the data. 
Conclusion: In this study, the pooled prevalence of prelacteal feeding is high. Both individual and community level variables were associated with prelacteal feeding practice. Therefore, individual and community-level interventions that encourage mothers to deliver in the health facility and promoting timely initiation of breastfeeding are needed to reduce prelacteal feeding practices in east Africa. Moreover, media campaigns regarding this harmful traditional practice could be strengthened.

Keywords: Prelacteal feeding, East Africa, Multilevel analysis

\section{Background}

Optimal breastfeeding is important for the immediate and long-lasting health of the child by preventing the most childhood killers: pneumonia and diarrhea [1]. Practicing optimal breastfeeding is very important for the prevention of undernutrition and the cognitive development of the newborn [2-4]. Moreover, optimal breastfeeding significantly reduces the risk of developing different infectious diseases and non-infectious inflammatory diseases such as allergy and asthma, as well as obesity and chronic non-communicable diseases such as diabetes mellitus $[5,6]$.

Initiating breastfeeding within $1 \mathrm{~h}$ of birth, exclusively breastfed for the first 6 months of life, and continued breastfeeding up to the age of 2 years are recommended by the World Health Organization and United Nations Children's Fund [1]. However, in different countries including countries in East Africa, the majority of mothers offer suboptimal breastfeeding practices to their newborns [7-16].

Prelacteal feeding is giving foods or liquids (except recommended medications) to newborns before breastfeeding is established [1]. It is a major public health problem that increases the risk of acquiring respiratory tract infections, diarrhea, and malnutrition [5, 17]. Furthermore, the practice of prelacteal feeding deprives newborns of taking colostrum that is rich in nutrients and immunoglobulins $[18,19]$. It has also shown that giving prelacteal foods delays breastfeeding initiation and interferes with exclusive breastfeeding $[1,3,20,21]$.

Despite its great effects on the health of the newborn, prelacteal feeding is widely practiced in many countries in the world with the highest prevalence in the southeast and central Asia, and Latin America [7, 21-23]. In Africa, most of mothers provide prelacteal foods to their newborn, and in sub-Saharan Africa, about 32.2\% newborns are exposed to prelacteal foods [15, 24, 25]. Works of the literature revealed that maternal education [22, 26, 27], antenatal care (ANC) utilization [15, 27], home delivery $[13,28]$, delivery by cesarean section $[14,22$, 27 , sex of the child $[15,28]$, and late initiation of breastfeeding [28] are among the factors that are associated with prelacteal feeding practice.

Although numerous studies are done on prelacteal feeding practice in individual east African countries, most of them did not consider the community-level factors that could affect the likelihood of prelacteal feeding. Therefore, we aimed to assess the pooled prevalence and associated factors of prelacteal feeding practice in east Africa. Identifying various factors at both individual and community levels can have a key role in implementing policies and programs aimed at minimizing prelacteal feeding practices.

\section{Methods}

Data source, data collection, and study population

We used pooled data from the 12 east Africa countries Demographic and Health Surveys (DHS) that were conducted from 2008 to 2019. All these surveys used a stratified two-stage cluster sampling technique. The key demographic and health indicators were collected in each DHS [29]. Five questionnaires: the Household Questionnaire, the Woman's Questionnaire, Man's Questionnaire, the Biomarker Questionnaire, and the Health Facility questionnaire were used in each survey to collect the demographic and health indicators. A pretest was performed before collecting the data and a debriefing session was held with the pre-test field staff [30]. Further information regarding the data collection procedure is found in each countries survey report.

For our study, we used a kid's data set with a total weighted sample of 33,423 women (Fig. 1).

\section{Variables of the study \\ Dependent variable}

The outcome variable was prelacteal feeding practice, which is defined as giving anything other than breast milk for the newborn in the first 3 days after delivery [31].

\section{Independent variables}

Both individual and community level explanatory variables were incorporated in this study (Table 1).

Individual-level variables Women age, educational level, maternal occupation, wealth quantile, ANC visit, place of delivery, delivery by cesarean section, the timing of initiation of breastfeeding, perception of distance from the health facility, media exposure, parity, sex of the child, birth order, and size of the child at birth. 


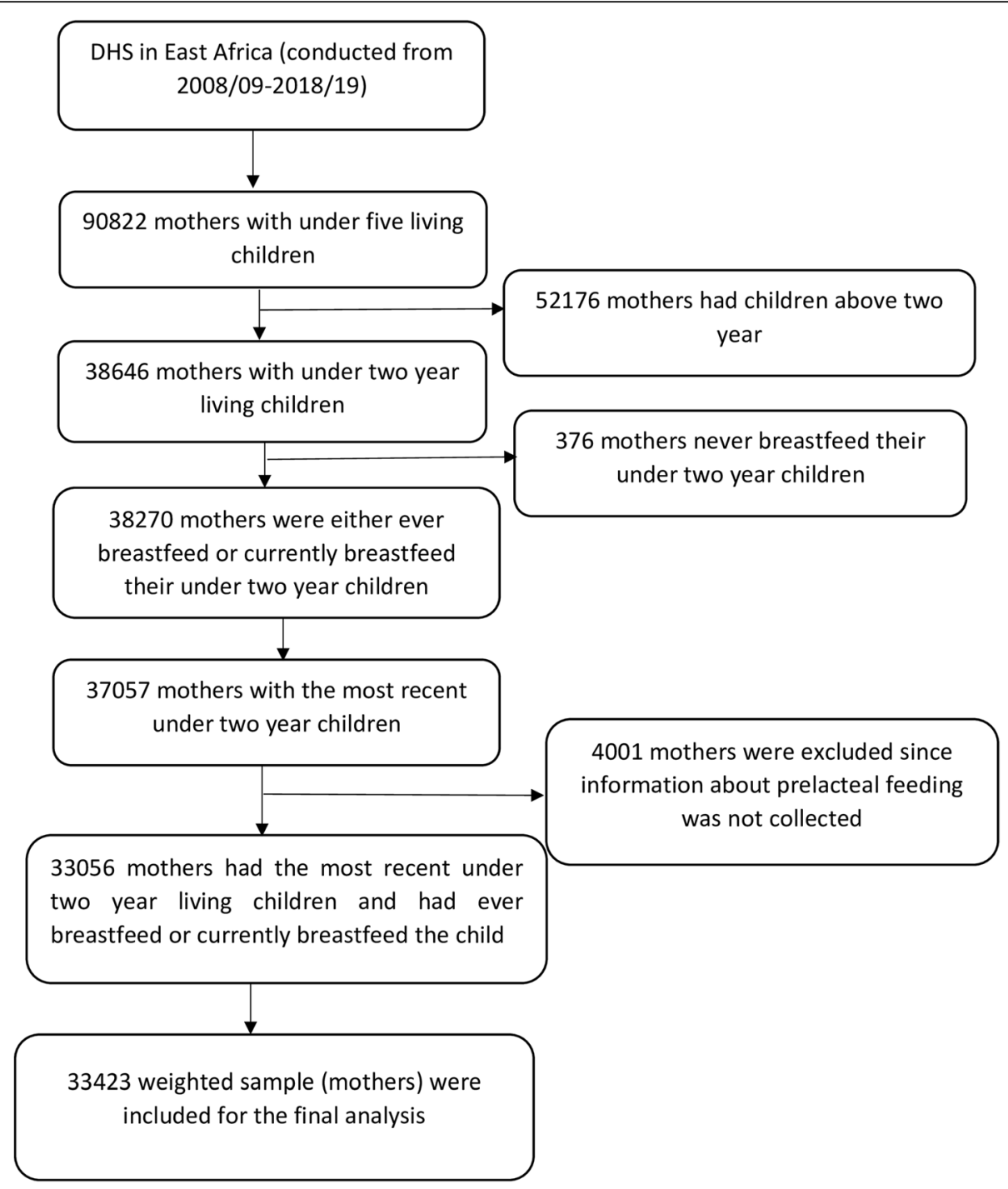

Fig. 1 Schematic presentation of how the study sample was selected

Community-level variables In this study, place of residence was a non-aggregate community-level variable while community level of women education, community level of media exposure, community level of ANC utilization, and community poverty level were constructed through the aggregation of individual-level factors to conceptualize their neighborhood effect on prelacteal feeding practice.

\section{Data management and statistical analysis}

The data were appended, recoded, and analyzed using Stata 14 software. The sample was weighted using the primary sampling unit variable, stratification variable, and the weight variable, to restore its representativeness and to get a better estimate throughout the analysis [32]. Both the weighted and unweighted results were presented and compared. The proportion of prelacteal feeding practice per each independent variable and the absolute risk difference was calculated. The pooled data have a hierarchical structure with individuals nested within clusters. Therefore, we employed a multilevel logistic regression analysis. To conduct the multilevel logistic regression analysis, four models were fitted. These are: the null model (a model containing only the outcome variable), model 1 (a model with the outcome variable and individual-level variables), model 2 (a model with the outcome variable and the community variables only), and model 3 (a model with the outcome variable and both the individual and community level variables).

The random effect analysis that is a measure of variation of prelacteal-feeding practice across communities or clusters, were assessed using intra-class correlation coefficient (ICC), median odds ratio (MOR), and a proportional change in variance (PCV) [33-35]. Since the 
Table 1 Description and categorization of independent variables

\begin{tabular}{|c|c|}
\hline Variables & Description/categorization of independent variables \\
\hline \multicolumn{2}{|l|}{ Individual-level variables } \\
\hline Women age & $\begin{array}{l}\text { It was the current age of the mother and grouped into seven categories: 15-19 years, 20-24 years, 25-29 years, 30-34 years, 35-39 } \\
\text { years, } 40-44 \text { years, and } 45-49 \text { years. }\end{array}$ \\
\hline Educational level & $\begin{array}{l}\text { It was the minimum level of education the mother achieve and it was coded as no formal education, primary education, and } \\
\text { secondary and above (combining secondary and higher education categories). }\end{array}$ \\
\hline Mother's occupation & It was the current working status of a woman, which was grouped into non-working and working. \\
\hline Wealth quantile & $\begin{array}{l}\text { In the DHS, it was based on wealth quintiles, with the first (lowest) quintile being the poorest, second (poorer), middle, fourth } \\
\text { (rich), and the highest quintile being the richest. For our study, it was re-categorized as poor (includes the lowest and the second } \\
\text { wealth quantiles), middle, and rich (includes the fourth and the highest wealth quantiles). }\end{array}$ \\
\hline ANC visit & $\begin{array}{l}\text { It was defined as the number of ANC visits while a mother was pregnant and it was coded as no ANC visit, 1-2 ANC visits, } 3 \text { ANC } \\
\text { visits, and } 4 \text { and above ANC visits. }\end{array}$ \\
\hline Place of delivery & Grouped into delivery at home and health facility. \\
\hline Delivery by cesarean section & Categorized as yes (if the mother gave birth by CS) and no (otherwise). \\
\hline $\begin{array}{l}\text { Timing of initiation of } \\
\text { breastfeeding }\end{array}$ & $\begin{array}{l}\text { It was the time at which the mother gave breast milk to the newborn baby and categorized as early (at/within } 1 \mathrm{~h} \text { ) and delayed } \\
\text { (after } 1 \mathrm{~h} \text { ). }\end{array}$ \\
\hline $\begin{array}{l}\text { Perception of distance from the } \\
\text { health facility }\end{array}$ & It was based on whether the mother perceives distance from the health facility as a big problem or not a big problem \\
\hline media exposure & $\begin{array}{l}\text { Created by combining whether a respondent reads a newspaper, listens to the radio, and watches television and coded as yes (if } \\
\text { a woman had been exposed to at least one of these media) and no (otherwise). }\end{array}$ \\
\hline Parity & $\begin{array}{l}\text { Categorized as Primiparous (having parity of one), multiparous (having parity of 2-4), and grand multiparous (having parity of } 5 \\
\text { and above). }\end{array}$ \\
\hline Multiple birth & It was coded as no (if women gave a single birth) and yes (otherwise) \\
\hline Sex of the child & The sex of the child was categorized into female and male. \\
\hline Size of the child at birth & $\begin{array}{l}\text { It was based on the mother's perception and categorized as very small, smaller than average, average, larger than average, and } \\
\text { very large in the DHS. This variable was re-categorized into small (very small, smaller than average), average (average), and large } \\
\text { (larger than average and very large) for our study. }\end{array}$ \\
\hline \multicolumn{2}{|l|}{ Community-level variables } \\
\hline Residence & The place of residence for the mother and coded as rural and urban \\
\hline $\begin{array}{l}\text { Community-level women } \\
\text { education }\end{array}$ & $\begin{array}{l}\text { It was the proportion of women with a minimum of primary level of education derived from data on mothers or respondent's } \\
\text { level of education. Then, it was categorized using national median value to values: low (if the mother was from communities in } \\
\text { which } \leq 50 \% \text { of women had at least primary education) and high (if the mother was from communities in which }>50 \% \text { of women } \\
\text { had at least primary education) community educational level. }\end{array}$ \\
\hline Community poverty level & $\begin{array}{l}\text { It was the proportion of women in the poorest and poorer quintiles derived from data on the household wealth index. Then, it } \\
\text { was categorized, based on national median value, into: low (if the mother was from communities in which } \leq 50 \% \text { of women had } \\
\text { poor socioeconomic status) and high (if the mother was from communities in which }>50 \% \text { of women had poor socioeconomic } \\
\text { status) community poverty level. }\end{array}$ \\
\hline $\begin{array}{l}\text { Community-level of ANC } \\
\text { utilization }\end{array}$ & $\begin{array}{l}\text { It was the proportion of women with at least one ANC visit and categorized using national level quartiles to: low (if the mother } \\
\text { was from communities in which } \leq 25 \% \text { of women utilizing ANC), middle (if the mother was from communities in which } 25-75 \% \text { of } \\
\text { women utilizing ANC), and high (if the mother was from communities in which } \geq 75 \% \text { of women utilizing ANC) community-level } \\
\text { ANC utilization. }\end{array}$ \\
\hline Community-level media exposure & $\begin{array}{l}\text { The proportions of mothers who were exposed to media within a specific cluster. It was categorized in the same fashion as the } \\
\text { community level of women's education into low and high community-level of media exposure. }\end{array}$ \\
\hline
\end{tabular}

Note: $\mathrm{ANC}=$ Antenatal Care, $\mathrm{CS}=$ Cesarean Section, DHS=Demographic and Health Surveys

models fitted are nested models, deviance was used for model comparison and model fitness.

Both bivariable and multivariable multilevel analyses were done and variables with $p$-value $<0.20$ in the bivariable analysis were eligible for multivariable analysis. Finally, the Adjusted Odds Ratio (AOR) with 95\% Confidence Interval (CI) was reported and variables with $p$ value $<0.05$, in the multivariable analysis, were declared to be significant predictors of prelacteal feeding practice. Variance inflation factor (VIF) was used to test Multicollinearity and there was no Multicollinearity between independent variables.

\section{Results}

\section{Socio-demographic characteristics of the study population}

Among 38,270 mothers, with under 2 year living children, who ever breastfeed or are breastfeed their child, 33,423 mothers (weighted) were included for the final analysis (Fig. 1). The majority of the study participants were from Mozambique, Kenya, Tanzania, Zambia, and Ethiopia. Regarding place of residence, more than three fourth $(77.23 \%)$ of respondents were rural dwellers. The median age of mothers was $27(\mathrm{IQR} \pm 10)$ years. About half $(50.36 \%)$ of the respondents had a primary level of education and $45.83 \%$ of respondents were from 
households with poor socioeconomic status. Regarding the timing of breastfeeding initiation, the majority $(80.67 \%)$ of respondents initiate breast milk within $1 \mathrm{~h}$. More than half (51.7\%) and three-fourth (78.86\%) of respondents had four and above ANC visits and gave their last birth at the health facility, respectively (Table 2).

\section{Proportion of prelacteal feeding practice by socio- demographic characteristics and the absolute risk difference}

Table 3 revealed the weighted and unweighted proportion of prelacteal feeding by each independent variable and their absolute risk difference. The weighted percentage of prelacteal feeding among mothers who gave a multiple birth was $20.23 \%$ while in those who gave single birth was $11.73 \%$ with an absolute risk difference of $8.56 \%$. The proportion of prelacteal feeding among mothers who initiated breast milk within an hour and after an hour was 7.99 and $27.93 \%$, respectively, with an absolute risk difference of $19.94 \%$. The absolute risk difference of prelacteal feeding among those mothers who gave birth at home and at the health facility was $8.46 \%$. Regarding country, the highest absolute risk difference (36.13\%) was found between Comoros and Malawi (Table 3).

\section{Prevalence of prelacteal feeding practice in East Africa}

The prevalence of prelacteal feeding practice based on the weighted data was $11.85 \%$ (95\% CI: $11.50,12.20$ ) with great variation between countries (Fig. 2). However, using the unweighted data, the pooled prevalence was 12.83\% (95\%CI: 12.48, 13.20) (Fig. 3).

\section{Factors associated with prelacteal feeding practice in East Africa \\ Fixed effect analysis}

Table 4 revealed a multilevel analysis for the final model, both for the weighted and unweighted data. We considered the weighted data to assess factors associated with prelacteal feeding practice in East Africa since it gives an appropriate parameter estimate to draw a valid conclusion. Therefore, the interpretations here are based on the weighted data. In the multivariable multilevel analysis; multiple birth, the timing of breastfeeding initiation, media exposure, place of delivery, delivery by cesarean section, size of the child at birth, residence, and community level of media exposure were significantly associated with prelacteal feeding practice $(p<0.05)$. Mothers who gave a multiple birth had 1.69 [AOR = 1.69; 95\% CI: 1.22, 1.34] times higher odds of prelacteal feeding practice compared to their counterparts. The odds of practicing prelacteal feeding practice was 3.83 $[\mathrm{AOR}=3.48 ; 95 \% \mathrm{CI}: 3.48,4.23]$ times higher among mothers who initiated breastfeeding after $1 \mathrm{~h}$ compared to their counterparts. Regarding media exposure, mothers who had not been exposed to at least one media had 1.21 [AOR $=1.21$; 95\% CI: $1.07,1.35$ ] times higher odds of prelacteal feeding practice as compared to those who had exposed to at least one media. Mothers who gave birth in the health facility had 56\% [AOR $=0.44 ; 95 \%$ CI: $0.39,0.49$ ] lower odds of prelacteal feeding practice as compared to those who delivered at home. Delivery by cesarean section was also associated with prelacteal feeding in which the odds of prelacteal feeding practice was 1.63 [AOR $=1.63 ; 95 \%$ CI: 1.38 , 1.93] times higher among mothers who delivered by cesarean section as compared to those who gave vaginal birth. The odds of prelacteal feeding practice was 1.15 [AOR $=1.15 ; 95 \%$ CI: 1.01, 1.32] times higher among mothers who gave a small-sized baby as compared to those mothers who gave a large-sized baby. Mothers from the rural area had 22\% [AOR $=0.78 ; 95 \%$ CI: 0.67 , 0.91 l lower odds of prelacteal feeding practice as compared to those from urban areas. Regarding communitylevel of media exposure, mothers from communities with a lower level of media exposure had 1.22 [AOR = 1.22; 95\% CI: 1.09, 1.36] times higher odds of prelacteal feeding practice as compared to their counterparts (Table 4).

\section{Random effect analysis and model comparison}

Table 5 revealed the random effect analysis for the model with the weighted data. The ICC value in the null model indicates $9.3 \%$ of the total variations of prelacteal feeding practice were due to the difference between clusters. Besides, the high MOR value in the null model which was 1.74 revealed that when we randomly select mothers from two clusters, mothers from a high-risk cluster had 1.74 times more likely to practice prelacteal feeding as compared to mothers from a low-risk cluster. Moreover, the PCV in the final model revealed that about $13.4 \%$ of the variability in prelacteal feeding practice was explained both by individual and communitylevel factors. Regarding model fitness, model 3 was the best-fit model since it had the lowest deviance (Table 5).

\section{Discussion}

This study aimed to assess the pooled prevalence and associated factors of prelacteal feeding practice in east Africa. The pooled prevalence of prelacteal feeding was $12 \%$. The prevalence in this study is in line with a study done in Ethiopia [36], however, it is lower than reports from other studies [7, 8, 37-39] (Table 6). This discrepancy might be due to the difference in the study population, the variation in living conditions, and the difference in access to media and information across countries. This suggests strategies concerning 
Table 2 Sociodemographic characteristics of respondents and their children

\begin{tabular}{|c|c|c|c|c|}
\hline Variables & Unweighted frequency $(N=33,056)$ & Percentage (\%) & $\begin{array}{l}\text { Weighted frequency } \\
(N=33,423)\end{array}$ & Percentage (\%) \\
\hline \multicolumn{5}{|l|}{ Country } \\
\hline Burundi & 2504 & 7.58 & 2579 & 7.72 \\
\hline Ethiopia & 3821 & 11.56 & 4050 & 12.12 \\
\hline Kenya & 3659 & 11.07 & 3386 & 10.13 \\
\hline Comoros & 1088 & 3.29 & 1129 & 3.38 \\
\hline Madagascar & 2326 & 7.04 & 2374 & 7.10 \\
\hline Malawi & 2141 & 6.48 & 2131 & 6.37 \\
\hline Mozambique & 4247 & 12.83 & 4549 & 13.61 \\
\hline Rwanda & 1481 & 4.48 & 1507 & 4.51 \\
\hline Tanzania & 4002 & 12.11 & 3956 & 11.84 \\
\hline Uganda & 1847 & 5.59 & 1807 & 5.41 \\
\hline Zambia & 3757 & 11.37 & 3691 & 11.04 \\
\hline Zimbabwe & 2183 & 6.60 & 2264 & 6.77 \\
\hline \multicolumn{5}{|c|}{ Maternal age (years) } \\
\hline $15-19$ & 3657 & 11.06 & 3734 & 11.17 \\
\hline $20-24$ & 8864 & 26.82 & 8824 & 26.40 \\
\hline $25-29$ & 8371 & 25.32 & 8522 & 25.50 \\
\hline $30-34$ & 6218 & 18.81 & 6299 & 18.85 \\
\hline $35-39$ & 4037 & 12.21 & 4104 & 12.28 \\
\hline $40-44$ & 1595 & 4.83 & 1610 & 4.82 \\
\hline $45-49$ & 314 & 0.95 & 330 & 0.99 \\
\hline \multicolumn{5}{|l|}{ Educational level } \\
\hline No education & 8456 & 25.58 & 8467 & 25.33 \\
\hline Primary & 16,222 & 49.07 & 16,831 & 50.36 \\
\hline Sec \& above & 8378 & 25.34 & 8125 & 24.31 \\
\hline \multicolumn{5}{|c|}{ Maternal occupation } \\
\hline Working & 20,829 & 63.01 & 21,449 & 64.17 \\
\hline Not working & 12,227 & 36.99 & 11,974 & 35.83 \\
\hline \multicolumn{5}{|l|}{ Wealth index } \\
\hline Poor & 15,441 & 46.71 & 15,316 & 45.83 \\
\hline Middle & 6047 & 18.29 & 6536 & 19.56 \\
\hline Rich & 11,568 & 35.00 & 11,571 & 34.62 \\
\hline \multicolumn{5}{|l|}{ Sex of child } \\
\hline Male & 16,522 & 49.98 & 16,743 & 50.09 \\
\hline Female & 16,534 & 50.02 & 16,680 & 49.91 \\
\hline \multicolumn{5}{|l|}{ Multiple birth } \\
\hline No & 32,578 & 98.55 & 32,963 & 98.62 \\
\hline Yes & 478 & 1.45 & 460 & 1.38 \\
\hline \multicolumn{5}{|c|}{ Breastfeeding initiation } \\
\hline Within $1 \mathrm{~h}$ & 26,642 & 80.60 & 26,963 & 80.67 \\
\hline After $1 \mathrm{~h}$ & 6414 & 19.40 & 6460 & 19.33 \\
\hline \multicolumn{5}{|l|}{ Media exposure } \\
\hline No & 12,167 & 36.81 & 12,071 & 36.12 \\
\hline
\end{tabular}


Table 2 Sociodemographic characteristics of respondents and their children (Continued)

\begin{tabular}{|c|c|c|c|c|}
\hline Variables & Unweighted frequency $(N=33,056)$ & Percentage (\%) & $\begin{array}{l}\text { Weighted frequency } \\
(N=33,423)\end{array}$ & Percentage (\%) \\
\hline Yes & 20,889 & 63.19 & 21,352 & 63.88 \\
\hline \multicolumn{5}{|l|}{ Parity } \\
\hline Primiparous & 6876 & 20.18 & 7021 & 21.01 \\
\hline Multiparous & 16,693 & 50.50 & 16,812 & 50.30 \\
\hline Grand multiparous & 9487 & 28.70 & 9590 & 28.69 \\
\hline \multicolumn{5}{|l|}{ ANC visits } \\
\hline No & 2449 & 7.41 & 2678 & 8.01 \\
\hline One \& two & 4284 & 12.96 & 4378 & 13.10 \\
\hline Three & 8976 & 27.15 & 9074 & 27.15 \\
\hline Four \& above & 17,347 & 52.48 & 17,293 & 51.74 \\
\hline \multicolumn{5}{|l|}{ Place of delivery } \\
\hline Home & 9056 & 27.40 & 9072 & 27.14 \\
\hline Health facility & 24,000 & 72.60 & 24,351 & 72.86 \\
\hline \multicolumn{5}{|l|}{ Cesarean delivery } \\
\hline Yes & 1966 & 5.95 & 1951 & 5.84 \\
\hline No & 31,090 & 94.05 & 31,472 & 94.16 \\
\hline \multicolumn{5}{|c|}{ Size of the child at birth } \\
\hline Small & 5569 & 16.85 & 5522 & 16.52 \\
\hline Large & 9837 & 29.76 & 10,217 & 30.57 \\
\hline Average & 17,650 & 53.39 & 17,684 & 52.91 \\
\hline \multicolumn{5}{|c|}{ Distance from the health facility } \\
\hline Big problem & 13,998 & 42.35 & 14,769 & 44.19 \\
\hline Not a big problem & 19,058 & 57.65 & 18,654 & 55.81 \\
\hline \multicolumn{5}{|l|}{ Residence } \\
\hline Urban & 8318 & 25.16 & 7610 & 22.77 \\
\hline Rural & 24,738 & 74.84 & 25,813 & 77.23 \\
\hline \multicolumn{5}{|c|}{ Community-level women education } \\
\hline Low & 16,565 & 50.51 & 17,369 & 51.97 \\
\hline High & 16,491 & 49.89 & 16,054 & 48.03 \\
\hline \multicolumn{5}{|c|}{ Community poverty leve } \\
\hline Low & 16,272 & 49.23 & 17,208 & 51.48 \\
\hline High & 16,784 & 50.77 & 16,215 & 48.52 \\
\hline \multicolumn{5}{|c|}{ Community-level of ANC utilization } \\
\hline High & 7392 & 22.36 & 7044 & 21.08 \\
\hline Low & 11,312 & 34.22 & 12,258 & 36.67 \\
\hline Middle & 14,352 & 43.42 & 14,121 & 42.25 \\
\hline \multicolumn{5}{|c|}{ Community-level media exposure } \\
\hline Low & 16,255 & 49.17 & 16,047 & 48.01 \\
\hline High & 16,801 & 50.83 & 17,376 & 51.99 \\
\hline
\end{tabular}

Note: ANC Antenatal Care, sec Secondary

suboptimal feeding patterns are decreased over time due to the expanded utilization of maternal health services.

This study also found the high heterogeneity, from 3\% in Malawi to 39\% in Comoros, of prelacteal feeding practice across east African countries. This may be due to the difference in the study period. For example, the data for Comoros was collected in 2012, while the data for Malawi was collected in 2015. Besides, the high 
Table 3 Proportion of prelacteal feeding by each sociodemographic characteristic and the absolute risk difference

\begin{tabular}{|c|c|c|c|c|c|c|c|c|}
\hline \multirow[t]{2}{*}{ Variables } & \multicolumn{2}{|c|}{ PF (unweighted) } & \multicolumn{2}{|c|}{ PF (weighted) } & \multirow{2}{*}{$\begin{array}{l}\text { Unweighted } \\
\text { Proportion } \\
\text { of PF (\%) }\end{array}$} & \multirow{2}{*}{$\begin{array}{l}\text { ARD } \\
(\%)\end{array}$} & \multirow{2}{*}{$\begin{array}{l}\text { Weighted } \\
\text { Proportion } \\
\text { of PF (\%) }\end{array}$} & \multirow{2}{*}{$\begin{array}{l}\text { ARD } \\
\text { (\%) }\end{array}$} \\
\hline & No & Yes & No & Yes & & & & \\
\hline \multicolumn{9}{|l|}{ Maternal age (years) } \\
\hline $15-19$ & 3152 & 505 & 3236 & 498 & 13.81 & Ref. & 13.33 & Ref. \\
\hline $20-24$ & 7720 & 1144 & 7779 & 1045 & 12.91 & 0.90 & 11.84 & 1.47 \\
\hline $25-29$ & 7362 & 1009 & 7571 & 951 & 12.05 & 1.76 & 11.15 & 2.18 \\
\hline $30-34$ & 5408 & 810 & 5576 & 723 & 13.03 & 0.78 & 11.48 & 1.85 \\
\hline $35-39$ & 3511 & 526 & 3610 & 494 & 13.03 & 0.78 & 12.48 & 0.85 \\
\hline $40-44$ & 1390 & 205 & 1402 & 208 & 12.85 & 0.96 & 12.04 & 1.29 \\
\hline $45-49$ & 271 & 43 & 289 & 41 & 13.69 & 0.12 & 12.91 & 0.42 \\
\hline \multicolumn{9}{|l|}{ Educational level } \\
\hline No education & 8392 & 1360 & 7400 & 1067 & 16.08 & Ref. & 12.60 & Ref. \\
\hline Primary & 14,407 & 1815 & 14,969 & 1862 & 11.19 & 4.89 & 11.06 & 1.54 \\
\hline Sec \& above & 7311 & 1007 & 7094 & 1030 & 12.74 & 3.34 & 12.68 & -0.80 \\
\hline \multicolumn{9}{|l|}{ Mother's occupation } \\
\hline Working & 18,079 & 2750 & 18,695 & 2754 & 12.20 & Ref. & 12.84 & Ref. \\
\hline Not working & 10,735 & 1492 & 10,768 & 1206 & 13.20 & 1.00 & 10.07 & 2.77 \\
\hline \multicolumn{9}{|l|}{ Wealth index } \\
\hline Poor & 13,295 & 2146 & 13,436 & 1880 & 13.90 & Ref. & 12.27 & Ref. \\
\hline Middle & 5357 & 690 & 5798 & 738 & 11.41 & 2.49 & 11.29 & 0.98 \\
\hline Rich & 10,162 & 1406 & 10,230 & 1341 & 12.15 & 1.75 & 11.59 & 0.68 \\
\hline \multicolumn{9}{|l|}{ Sex of child } \\
\hline Male & 14,348 & 2174 & 14,709 & 2034 & 13.16 & Ref. & 12.14 & Ref. \\
\hline Female & 14,466 & 2068 & 14,754 & 1926 & 12.51 & 0.65 & 11.55 & 0.59 \\
\hline \multicolumn{9}{|l|}{ Multiple birth } \\
\hline No & 28,433 & 4145 & 29,097 & 3866 & 12.72 & Ref. & 11.73 & Ref. \\
\hline Yes & 381 & 97 & 367 & 93 & 20.29 & -7.57 & 20.29 & -8.56 \\
\hline \multicolumn{9}{|l|}{ Breastfeeding initiation } \\
\hline Within $1 \mathrm{~h}$ & 24,319 & 2323 & 24,808 & 2155 & 8.71 & Ref. & 7.99 & Ref. \\
\hline After $1 \mathrm{~h}$ & 4495 & 1919 & 4656 & 1804 & 29.92 & -21.21 & 27.93 & -19.94 \\
\hline \multicolumn{9}{|l|}{ Media exposure } \\
\hline No & 10,606 & 1561 & 10,772 & 1299 & 12.83 & Ref. & 10.76 & Ref. \\
\hline Yes & 18,208 & 2681 & 18,691 & 2661 & 12.83 & 0.00 & 12.46 & -1.70 \\
\hline \multicolumn{9}{|l|}{ Parity } \\
\hline Primiparous & 5887 & 989 & 6070 & 951 & 14.38 & Ref. & 13.55 & Ref. \\
\hline Multiparous Grand & 14,712 & 1972 & 14,977 & 1835 & 11.81 & 2.57 & 10.91 & 2.64 \\
\hline multiparous & 8206 & 1281 & 8417 & 1173 & 13.50 & 0.88 & 12.24 & 1.31 \\
\hline \multicolumn{9}{|l|}{ ANC visits } \\
\hline No & 1890 & 559 & 2258 & 420 & 22.83 & Ref. & 15.69 & Ref. \\
\hline One \& two & 3631 & 653 & 3793 & 585 & 15.24 & 7.59 & 13.35 & 2.34 \\
\hline Three & 7956 & 1020 & 8074 & 1000 & 11.36 & 11.47 & 11.02 & 4.67 \\
\hline Four \& above & 15,337 & 2010 & 15,338 & 1955 & 11.59 & 11.24 & 11.30 & 4.39 \\
\hline \multicolumn{9}{|l|}{ Place of delivery } \\
\hline Home & 7173 & 1883 & 7438 & 1634 & 20.79 & Ref. & 18.01 & Ref. \\
\hline Health facility & 21,641 & 2359 & 22,026 & 2325 & 9.83 & 10.96 & 9.55 & 8.46 \\
\hline
\end{tabular}


Table 3 Proportion of prelacteal feeding by each sociodemographic characteristic and the absolute risk difference (Continued)

\begin{tabular}{|c|c|c|c|c|c|c|c|c|}
\hline \multirow[t]{2}{*}{ Variables } & \multicolumn{2}{|c|}{ PF (unweighted) } & \multicolumn{2}{|c|}{ PF (weighted) } & \multirow{2}{*}{$\begin{array}{l}\text { Unweighted } \\
\text { Proportion } \\
\text { of PF (\%) }\end{array}$} & \multirow{2}{*}{$\begin{array}{l}\text { ARD } \\
\text { (\%) }\end{array}$} & \multirow{2}{*}{$\begin{array}{l}\text { Weighted } \\
\text { Proportion } \\
\text { of PF (\%) }\end{array}$} & \multirow{2}{*}{$\begin{array}{l}\text { ARD } \\
\text { (\%) }\end{array}$} \\
\hline & No & Yes & No & Yes & & & & \\
\hline \multicolumn{9}{|l|}{ Cesarean delivery } \\
\hline Yes & 1531 & 435 & 1527 & 424 & 22.13 & -9.88 & 21.72 & -10.49 \\
\hline No & 27,283 & 3807 & 27,937 & 3535 & 12.25 & Ref. & 11.23 & Ref. \\
\hline \multicolumn{9}{|c|}{ Size of the child at birth } \\
\hline Small & 4637 & 932 & 4732 & 790 & 16.74 & -4.22 & 14.32 & -2.75 \\
\hline Average & 1557 & 2078 & 15,697 & 1987 & 11.77 & 0.75 & 11.23 & 0.34 \\
\hline Large & 8605 & 1232 & 9035 & 1182 & 12.52 & Ref. & 11.57 & Ref. \\
\hline \multicolumn{9}{|c|}{ Distance from the health facility } \\
\hline Big problem & 12,146 & 1852 & 13,005 & 1764 & 13.23 & Ref. & 11.95 & Ref. \\
\hline Not a big problem & 16,668 & 2390 & 16,459 & 2195 & 12.54 & 0.69 & 11.77 & 0.18 \\
\hline \multicolumn{9}{|l|}{ Residence } \\
\hline Urban & 7207 & 1111 & 6613 & 997 & 13.36 & Ref. & 13.10 & Ref. \\
\hline Rural & 21,607 & 3131 & 22,851 & 2962 & 12.66 & 0.70 & 11.48 & 1.62 \\
\hline \multicolumn{9}{|c|}{ Community-level women education } \\
\hline Low & 14,506 & 2059 & 15,396 & 1973 & 12.43 & Ref. & 11.36 & Ref. \\
\hline High & 14,308 & 2183 & 14,068 & 1986 & 13.24 & -0.81 & 12.37 & -1.01 \\
\hline \multicolumn{9}{|c|}{ Community poverty level } \\
\hline Low & 14,100 & 2172 & 15,096 & 2112 & 13.35 & Ref. & 12.27 & Ref. \\
\hline High & 14,714 & 2070 & 14,368 & 1847 & 12.33 & 1.02 & 11.39 & 0.88 \\
\hline \multicolumn{9}{|c|}{ Community-level of ANC utilization } \\
\hline High & 6430 & 962 & 6159 & 885 & 13.01 & Ref. & 12.56 & Ref. \\
\hline Low & 10,037 & 1275 & 10,980 & 1278 & 11.27 & 1.74 & 10.43 & 2.13 \\
\hline Middle & 12,347 & 2005 & 12,325 & 1796 & 13.97 & -0.96 & 12.72 & -0.16 \\
\hline \multicolumn{9}{|c|}{ Community-level media exposure } \\
\hline Low & 14,287 & 1968 & 14,342 & 1705 & 12.11 & 1.41 & 10.63 & 2.34 \\
\hline High & 14,527 & 2274 & 15,122 & 2254 & 13.53 & Ref. & 12.97 & Ref. \\
\hline \multicolumn{9}{|l|}{ Country } \\
\hline Burundi & 2349 & 155 & 2440 & 139 & 6.19 & 32.30 & 5.39 & 33.71 \\
\hline Ethiopia & 3199 & 622 & 3728 & 322 & 16.28 & 22.21 & 7.95 & 31.21 \\
\hline Kenya & 3134 & 525 & 2867 & 519 & 14.35 & 24.14 & 15.31 & 23.90 \\
\hline Comoros & 666 & 422 & 686 & 443 & 38.49 & Ref. & 39.21 & Ref. \\
\hline Madagascar & 1672 & 654 & 1757 & 617 & 28.12 & 10.37 & 26.00 & 13.21 \\
\hline Malawi & 2078 & 63 & 2065 & 66 & 2.94 & 35.35 & 3.08 & 36.13 \\
\hline Mozambique & 4009 & 238 & 4288 & 261 & 5.60 & 32.89 & 5.75 & 33.46 \\
\hline Rwanda & 1417 & 64 & 1441 & 66 & 4.32 & 34.17 & 4.34 & 34.87 \\
\hline 'Tanzania & 3404 & 598 & 3411 & 545 & 14.94 & 23.55 & 13.78 & 25.43 \\
\hline Uganda & 1406 & 441 & 1355 & 452 & 23.88 & 14.61 & 25.01 & 14.20 \\
\hline Zambia & 3544 & 213 & 3444 & 247 & 5.67 & 32.82 & 7.70 & 31.51 \\
\hline Zimbabwe & 1936 & 247 & 1981 & 283 & 11.31 & 27.18 & 12.49 & 26.72 \\
\hline
\end{tabular}




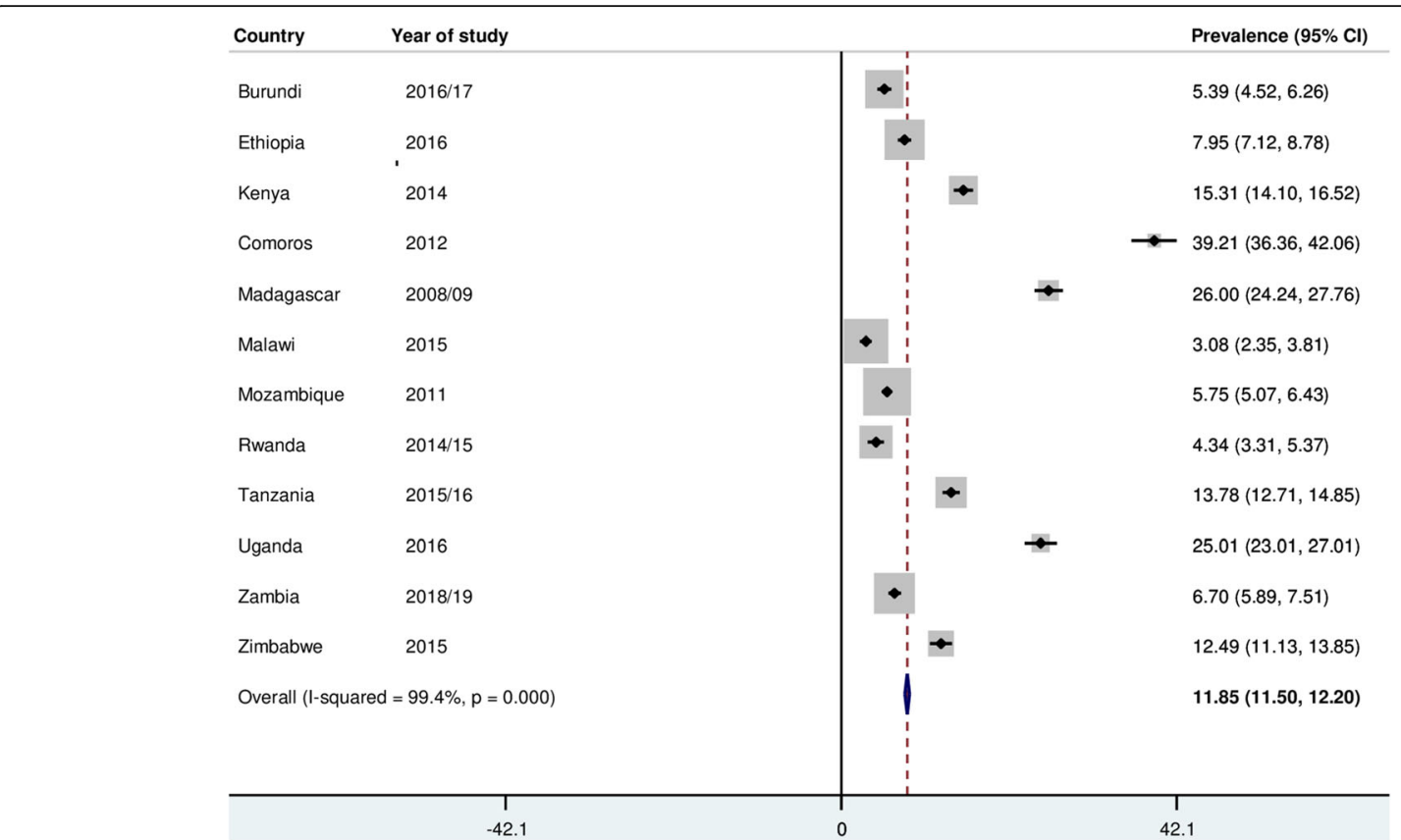

Fig. 2 The pooled prevalence of prelacteal feeding practice in East Africa (Weighted)

heterogeneity in prelacteal feeding practice may be due to the sociocultural and socioeconomic differences among mothers in east African countries.

This study identified different factors that were associated with prelacteal feeding practice. In the unweighted data analysis, factors such as maternal education, multiple birth, media exposure, parity, ANC visit, place of delivery, delivery by cesarean section, birth size, residence, community-level of media exposure, communitylevel of ANC utilization, and community poverty level were associated with prelacteal feeding practice.

However, weighed data analysis identified multiple birth, the timing of breastfeeding initiation, media exposure, place of delivery, delivery by cesarean section,

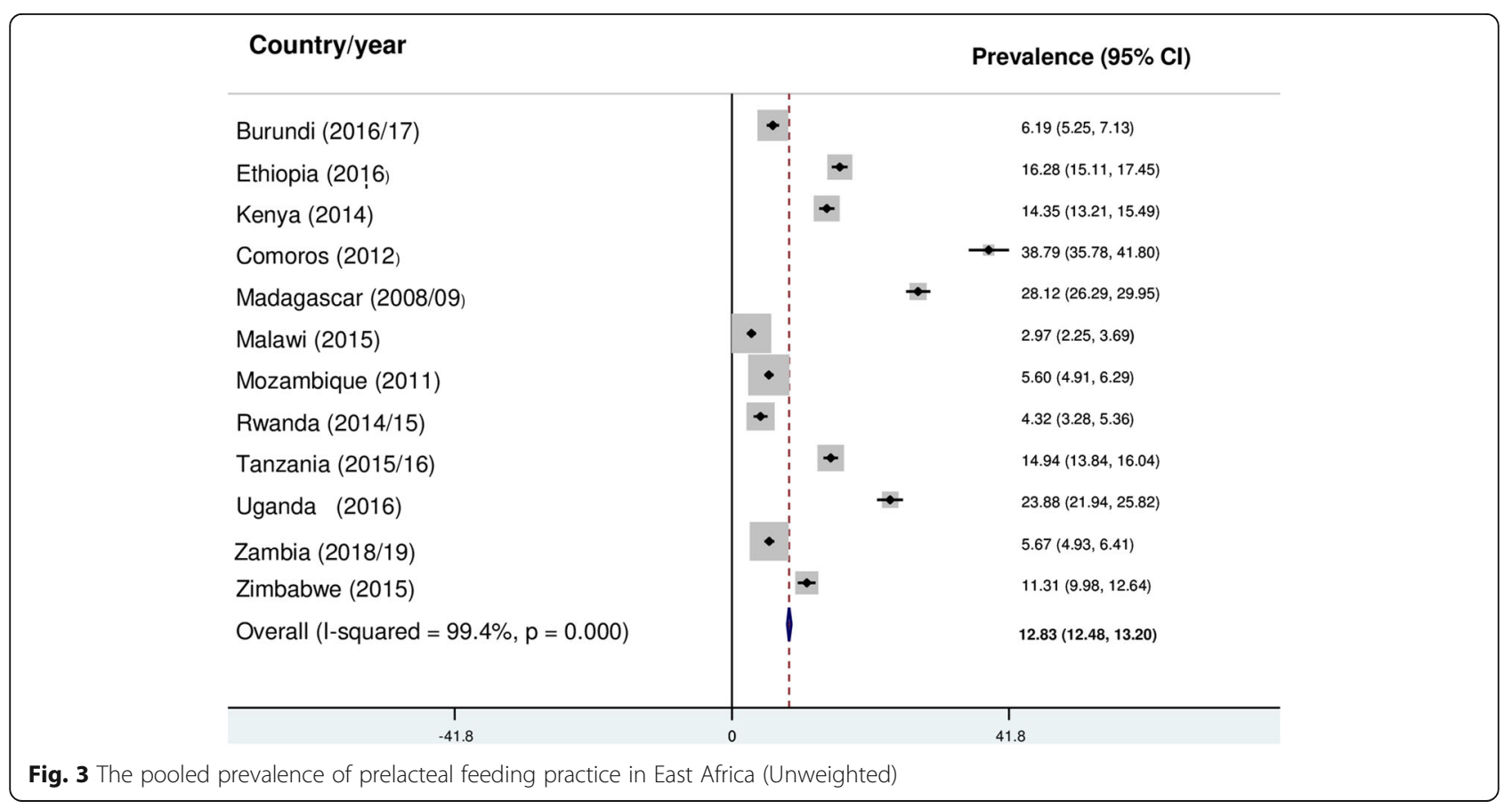


Table 4 Factors associated with prelacteal feeding practice in East Africa

\begin{tabular}{|c|c|c|c|c|}
\hline \multirow[t]{3}{*}{ Variables } & \multicolumn{4}{|c|}{ Multilevel Logistic regression } \\
\hline & \multicolumn{2}{|c|}{ Based on unweighted data } & \multicolumn{2}{|c|}{ Based on weighted data } \\
\hline & COR $(95 \% \mathrm{Cl})$ & AOR $(95 \% \mathrm{Cl})$ & COR $(95 \% \mathrm{Cl})$ & AOR $(95 \% \mathrm{Cl})$ \\
\hline \multicolumn{5}{|l|}{ Maternal age (years) } \\
\hline $15-19$ & 1.00 & 1.00 & 1.00 & 1.00 \\
\hline $20-24$ & $0.91(0.81,1.02)$ & $0.92(0.81,1.05)$ & $0.87(0.75,1.01)$ & $0.90(0.76,1.06)$ \\
\hline $25-29$ & $0.85(0.76,0.95)$ & $0.85(0.74,0.97) *$ & $0.82(0.70,0.95)$ & $0.85(0.71,1.02)$ \\
\hline $30-34$ & $0.93(0.82,1.05)$ & $0.92(0.79,1.08)$ & $0.84(0.73,0.98)$ & $0.87(0.72,1.05)$ \\
\hline $35-39$ & $0.93(0.82,1.07)$ & $0.90(0.76,1.07)$ & $0.90(0.75,1.08)$ & $0.92(0.73,1.16)$ \\
\hline $40-44$ & $0.92(0.77,1.10)$ & $0.90(0.73,1.12)$ & $0.97(0.78,1.21)$ & $0.99(0.76,1.29)$ \\
\hline $45-49$ & $0.98(0.70,1.38)$ & $0.92(0.64,1.34)$ & $0.94(0.61,1.47)$ & $0.93(0.59,1.48)$ \\
\hline \multicolumn{5}{|l|}{ Educational level } \\
\hline No education & 1.00 & 1.00 & 1.00 & 1.00 \\
\hline Primary & $0.66(0.51,0.72)$ & $0.76(0.70,0.83) * * *$ & $0.85(0.76,0.95)$ & $0.89(0.78,1.01)$ \\
\hline Sec \& above & $0.76(0.69,0.83)$ & $0.88(0.79,0.99)^{*}$ & $0.99(0.86,1.13)$ & $1.03(0.88,1.20)$ \\
\hline \multicolumn{5}{|l|}{ Wealth index } \\
\hline Poor & 1.00 & 1.00 & 1.00 & 1.00 \\
\hline Middle & $0.80(0.73,0.88)$ & $0.93(0.84,1.03)$ & $0.91(0.81,1.02)$ & $0.97(0.86,1.60)$ \\
\hline Rich & $0.85(0.79,0.92)$ & $0.91(0.82,1.01)$ & $0.93(0.84,1.04)$ & $0.87(0.76,1.01)$ \\
\hline \multicolumn{5}{|l|}{ Sex of child } \\
\hline Male & 1.00 & 1.00 & 1.00 & 1.00 \\
\hline Female & $0.94(0.88,1.01)$ & $0.96(0.89,1.03)$ & $0.94(0.87,1.03)$ & $0.95(0.87,1.05)$ \\
\hline \multicolumn{5}{|l|}{ Multiple birth } \\
\hline No & 1.00 & 1.00 & 1.00 & 1.00 \\
\hline Yes & $1.77(1.41,2.23)$ & $1.58(1.24,2.03)^{* * *}$ & $1.98(1.47,2.66)$ & $1.69(1.22,1.34) * *$ \\
\hline \multicolumn{5}{|l|}{ Breastfeeding initiation } \\
\hline Within $1 \mathrm{~h}$ & 1.00 & 1.00 & 1.00 & 1.00 \\
\hline After $1 \mathrm{~h}$ & $4.57(4.26,4.90)$ & $3.89(3.61,4.18)$ & $4.58(4.16,5.03)$ & $3.83(3.48,4.23) * * *$ \\
\hline \multicolumn{5}{|l|}{ Media exposure } \\
\hline No & $0.99(0.93,1.06)$ & $1.15(1.06,2.24)^{* *}$ & $1.18(1.06,1.30)$ & $1.21(1.07,1.35)^{* *}$ \\
\hline Yes & 1.00 & 1.00 & 1.00 & 1.00 \\
\hline \multicolumn{5}{|l|}{ Parity } \\
\hline Primiparous & 1.00 & 1.00 & 1.00 & 1.00 \\
\hline Multiparous & $0.80(0.73,0.87)$ & $0.88(0.80,0.98)^{*}$ & $0.78(0.70,0.86)$ & $0.89(0.78,1.01)$ \\
\hline Grand multiparous & $0.93(0.85,1.02)$ & $0.90(0.79,1.03)$ & $0.89(0.80,1.01)$ & $0.91(0.77,1.08)$ \\
\hline \multicolumn{5}{|l|}{ ANC visits } \\
\hline No & 1.00 & 1.00 & 1.00 & 1.00 \\
\hline One \& two & $0.60(0.53,0.69)$ & $0.77(0.67,0.88)^{* * *}$ & $0.76(0.62,0.93)$ & $0.89(0.72,1.11)$ \\
\hline Three & $0.43(0.39,0.49)$ & $0.65(0.56,0.74)^{* * *}$ & $0.60(0.49,0.74)$ & $0.81(0.65,1.01)$ \\
\hline Four \& above & $0.44(0.40,0.49)$ & $0.68(0.60,0.77)^{* * *}$ & $0.63(0.52,0.76)$ & $0.85(0.70,1.41)$ \\
\hline \multicolumn{5}{|l|}{ Place of delivery } \\
\hline Home & 1.00 & 1.00 & 1.00 & 1.00 \\
\hline Health facility & $0.41(0.38,0.44)$ & $0.43(0.40,0.47)^{* * *}$ & $0.46(0.41,0.51)$ & $0.44(0.39,0.49) * * *$ \\
\hline \multicolumn{5}{|l|}{ Cesarean delivery } \\
\hline No & 1.00 & 1.00 & 1.00 & 1.00 \\
\hline
\end{tabular}


Table 4 Factors associated with prelacteal feeding practice in East Africa (Continued)

\begin{tabular}{|c|c|c|c|c|}
\hline \multirow[t]{3}{*}{ Variables } & \multicolumn{4}{|c|}{ Multilevel Logistic regression } \\
\hline & \multicolumn{2}{|c|}{ Based on unweighted data } & \multicolumn{2}{|c|}{ Based on weighted data } \\
\hline & COR $(95 \% \mathrm{Cl})$ & AOR $(95 \% \mathrm{Cl})$ & COR $(95 \% \mathrm{Cl})$ & AOR $(95 \% \mathrm{Cl})$ \\
\hline Yes & $2.09(1.86,2.33)$ & $1.65(1.45,1.88)^{* * *}$ & $2.28(1.96,2.65)$ & $1.63(1.38,1.93)^{* * *}$ \\
\hline \multicolumn{5}{|l|}{ Size of the child at birth } \\
\hline Small & $1.40(1.28,1.54)$ & $1.14(1.03,1.26)^{*}$ & $1.31(1.15,1.48)$ & $1.15(1.01,1.32) *$ \\
\hline Large & 1.00 & 1.00 & 1.00 & 1.00 \\
\hline Average & $0.93(0.86,1.01)$ & $0.92(0.83,1.01)^{*}$ & $0.96(0.87,1.06)$ & $0.95(0.86,1.06)$ \\
\hline \multicolumn{5}{|c|}{ Distance from the health facility } \\
\hline Big problem & 1.00 & 1.00 & 1.00 & 1.00 \\
\hline Not a big problem & $0.94(0.88,1.01)$ & $1.02(0.94,1.09)$ & $0.97(0.88,1.06)$ & $0.98(0.90,1.08)$ \\
\hline \multicolumn{5}{|l|}{ Residence } \\
\hline Urban & 1.00 & 1.00 & $0.84(0.74,0.95)$ & 1.00 \\
\hline Rural & $0.93(0.86,1.01)$ & $0.84(0.74,0.90)^{* * *}$ & & $0.78(0.67,0.91)^{* *}$ \\
\hline \multicolumn{5}{|c|}{ Community-level women education } \\
\hline Low & 1.00 & 1.00 & 1.00 & 1.00 \\
\hline High & $1.10(1.01,1.18)$ & $1.10(1.01,1.21)$ & $1.14(1.02,1.27)$ & $1.10(0.98,1.24)$ \\
\hline \multicolumn{5}{|c|}{ Community poverty level } \\
\hline Low & 1.00 & 1.00 & 1.00 & 1.00 \\
\hline High & $0.89(0.82,0.97)$ & $0.90(0.82,0.99)^{*}$ & $0.90(0.81,1.01)$ & $0.95(0.84,1.06)$ \\
\hline \multicolumn{5}{|c|}{ Community-level of ANC utilization } \\
\hline High & 1.00 & 1.00 & 1.00 & 1.00 \\
\hline Low & $0.76(0.67,0.85)$ & $0.84(0.74,0.95)^{*}$ & $0.73(0.64,0.84)$ & $0.86(0.71,1.01)$ \\
\hline Middle & $1.08(0.97,1.20)$ & $1.10(0.99,1.23)$ & $1.01(0.89,1.15)$ & $1.14(1.01,1.30)$ \\
\hline \multicolumn{5}{|c|}{ Community-level media exposure } \\
\hline Low & $1.17(1.07,1.27)$ & $1.11(1.01,1.23)^{*}$ & $1.30(1.16,1.45)$ & $1.22(1.09,1.36)^{* *}$ \\
\hline High & 1.00 & 1.00 & 1.00 & 1.00 \\
\hline
\end{tabular}

size of the child at birth, residence, and community level of media exposure as predictors of prelacteal feeding practice. This finding is consistent with different studies done elsewhere (Table 6). We prefer to discuss the results we get from the weighted data, which is necessary when we analyze DHS data [32]. Weighting preserves the representativeness of data and it helps to get standard and appropriate statistical estimate (robust standard error) [32]. Therefore, we give stress to the findings from weighed analysis and the interpretations and discussions, in this paper, are based on the weighted data.

Institutional delivery was associated with lower odds of prelacteal feeding practice. This is consistent with studies done in Ethiopia, Nigeria, and Pakistan [26, 4042]. This might be justified as many health centers and hospitals ensure breastfeeding counseling during

Table 5 Community-level variability of prelacteal feeding practice and model comparison

\begin{tabular}{lllll}
\hline Parameter & Null model & Model 1 & Model 2 & Model 3 \\
\hline Community-level variance $[S E]$ & $0.341(0.036)$ & $0.302(0.031)$ & $0.329(0.034)$ & $0.295(0.031)$ \\
ICC & 0.093 & 0.084 & 0.091 & 0.082 \\
MOR & $1.74(1.65,1.85)$ & $1.69(1.60,1.78)$ & $1.72(1.64,1.83)$ & $1.67(1.59,1.77)$ \\
PCV (\%) & Reference & 11.4 & 3.5 & 13.5 \\
Model fitness & & & 23,840 & 21,792 \\
Deviance & 23,918 & 21,868 & & \\
\hline
\end{tabular}

Note; ICC Intraclass Correlation Coefficient, MOR Median Odds Ratio, SE Standard Error 
Table 6 Previous study findings on prelacteal-feeding practice

\begin{tabular}{ll}
\hline Authors & Title of the article \\
\hline $\begin{array}{ll}\text { Nguyen, } 2013 & \text { Prelacteal feeding practices in Vietnam: challenges and associate } \\
\text { f7] } & \text { factors } \\
\text { Berde, } 2013 & \text { Determinants of prelacteal feeding practices in urban and rural } \\
{[8]} & \text { Nigeria }\end{array}$
\end{tabular}

El-Gilany, $2014[10]$ Newborn first Feed and prelacteal feeds in Mansoura, Egypt

Khanal, 2016

[12]

Berde, 2017 Risk factors for prelacteal feeding in sub-Saharan Africa: a multi[15] level analysis of population data from twenty-two countries

Ogah, 2012 A cross-sectional study of prelacteal feeding practice among [20]

Agho, 2016 Trends and predictors of prelacteal feeding practices in Nigeria [26] (2003-2013)

Belachew, 2016 [27]

Individual and community-level factors associated with introduction of prelacteal feeding in Ethiopia

Gualu, 2016 Determinants of prelacteal feeding practice

[28] among postpartum mothers in Debre Markos town, Amhara regional state, Ethiopia, 2016

Tekaly, 2017 Prelacteal feeding practice and associated factors among mothers [36] having children less than 2 years of age in Aksum town, central Tigray, Ethiopia

Temesgen, Prelacteal feeding and associated factors in Ethiopia: systematic 2018 [37] review and meta-analysis

Amele, 2019 Prelacteal feeding practice and its associated factors among [38] mothers of children age less than 24 months old in Southern Ethiopia

\section{Findings}

Prevalence

$73.5 \%$

Urban

Nigeria (49.8\%) \&

Rural Nigeria (66.4\%)

$58 \%$

Mu

Factors associated with prelacteal feeding

Cesarean section (AOR: 2.94; 95\% Cl: 2.39, 3.61)

No education and primary educational status ((AOR: 1.48; 95\% Cl:1.07, 2.04 and AOR: 1.31; 95\% Cl: $1.02,1.69$, respectively)

Home delivery (AOR: 1.53; 95\% Cl:1.24, 1.89) Cesarean Delivery (AOR: 1.87; 95\% Cl:1.25, 2.80) Multiple birth (AOR: 2.37; 95\% Cl:1.14, 4.95) Maternal education (AOR: 1.5; 95\%Cl: 1.1, 2.3), Father's education secondary (AOR: 3.0; 95\%Cl: 1.7, 5.3); receiving $A N C$ visits at private clinics and no antenatal care; Caesarean section (AOR: 2.1; 95\%Cl: 1.2, 3.2); female babies (AOR: 1.7; 95\%Cl: 1.1, 3.2), and low birth weight (AOR: 4.2; 95\%Cl: 1.6, 11.2)

(30.6\%) Higher parity (AOR: $2.05 ; 95 \% \mathrm{Cl}$ : 1.18, 3.54), low birth weight (AOR: 1.97; 95\% Cl: 1.23, 3.16), a cesarean delivery (AOR: 3.70; 95\% Cl: 2.37, 5.80), and wealthy status (AOR: 2.49; 95\% Cl: 1.52, 4.06)

32.2\% Cesarean section (AOR: 2.25; $95 \%$ Cl: 2.06, 2.46) Other factors are also significantly associated with an increased likelihood of prelacteal feeding such as the mother's lower educational status, lower number of ANC visits, home delivery, multiple birth, male infant, and having a small-sized baby at birth. Besides, belonging to lower quintiles decrease the odds of prelacteal feeding.

Delay in initiating breastfeeding increases the prelacteal feeding practice

Mothers with no schooling (AOR: 1.65; 95\% Cl: 1.33, 2.03),

Younger mothers (aged 15-24 years), Mothers who delivered at home (AOR: 1.45; 95\% Cl: $1.23,1.71)$, and

Delivered by caesarean section (AOR: 1.91; 95\% Cl: $1.17,3.13)$

$28.92 \%$

Caesarean delivery (AOR: 1.87; 95\% Cl: 1.28, 2.73), and late initiation of breastfeeding (AOR: 5.32; 95\% Cl: 4.65, 6.09). Higher economic status (AOR: 0.72; $95 \% \mathrm{Cl}: 0.54,0.98)$, large birth size of child (AOR: 0.80; 95\% Cl: 0.68, 0.95), and high community ANC use (AOR: $58 ; 95 \% \mathrm{Cl}$ : 0.38, 0.87).

19.1\% Inability to read and write (AOR: $3.5 ; 95 \% \mathrm{Cl}: 1.14$ 10.75), giving birth to a male (AOR: $2.8 ; 95 \% \mathrm{Cl}$ : 1.23, 6.37), home delivery (AOR: 4.4; $95 \% \mathrm{Cl}$ : 1.78, 10.85)

10.1\% (95\% < 4 ANC visit (AOR: 10.55; 95\% Cl: 4.78, 23.40),

Cl: 7.3, 13\%). Cesarean section (AOR: 4.38; 95\% Cl:1.72, 11.12)

25.29\% (95\% ANC visit (AOR: 0.25; 95\% Cl: 0.09, 0.69),

Cl: 17.43,

Timely initiation of breastfeeding (AOR: 0.28; $95 \%$ 33.15)

Cl: $0.21,0.38)$

Urban residence (AOR: 0.47; 95\% Cl: 0.26, 0.86)

Home birth (AOR: 3.93; 95\% Cl: 2.17, 7.10)

(20.6\%) (95\% Extended family type (AOR: 10.64; 95\% Cl: 1.05 ,

Cl: 17.5, 24.4) 10.71)

Lack of breastfeeding counseling (AOR: 5.16; 95\% Cl: $1.76,15.13)$ and Mothers who avoid colostrum (AOR: $9.72 ; 95 \% \mathrm{Cl}$ : $3.46,27.30$ ) 
Table 6 Previous study findings on prelacteal-feeding practice (Continued)

\begin{tabular}{|c|c|c|c|}
\hline \multirow[t]{2}{*}{ Authors } & \multirow[t]{2}{*}{ Title of the article } & \multicolumn{2}{|l|}{ Findings } \\
\hline & & Prevalence & Factors associated with prelacteal feeding \\
\hline $\begin{array}{l}\text { Patel, } 2013 \\
\text { [39] }\end{array}$ & $\begin{array}{l}\text { Factors associated with } \\
\text { prelacteal feeding and timely } \\
\text { initiation of breastfeeding in } \\
\text { hospital-delivered infants in India }\end{array}$ & $16.9 \%$ & $\begin{array}{l}\text { Lower maternal education (AOR: } 2.13 ; 95 \% \mathrm{Cl} 1.06 \text {, } \\
4.35 \text { ), } \\
\text { Muslim religion (AOR: } 2.27 \text {; 95\% Cl: 1.18, 4.36), and } \\
\text { Delivery by cesarean section (AOR: } 2.56 ; 95 \% \mathrm{Cl} \text { : } \\
1.56,4.19 \text { ) }\end{array}$ \\
\hline $\begin{array}{l}\text { Wolde, } 2019 \\
\text { [40] }\end{array}$ & $\begin{array}{l}\text { Prelacteal feeding and associated factors among mothers having } \\
\text { children less than } 24 \text { months of age, in Mettu district, Southwest } \\
\text { Ethiopia }\end{array}$ & $\begin{array}{l}14.2 \%[95 \% \\
\text { Cl: } 12.0,17.0]\end{array}$ & $\begin{array}{l}\text { No maternal education (AOR: } 3.54 ; 95 \% \mathrm{Cl}: 1.7 \text {, } \\
6.98 \text { ), } \\
\text { Single ANC visits (AOR: } 6.87 ; 95 \% \mathrm{Cl}: 3.21,14.73 \text { ), } \\
\text { Home delivery (AOR: } 3.04 ; 95 \% \mathrm{Cl}: 1.60,5.75) \text { and } \\
\text { Cesarean delivery (AOR: } 4.27 ; 95 \% \mathrm{Cl}: 2.28,7.99 \text { ) }\end{array}$ \\
\hline $\begin{array}{l}\text { Argaw, } 2019 \\
{[41]}\end{array}$ & $\begin{array}{l}\text { Factors associated with prelacteal feeding practices in Debre } \\
\text { Berhan district, North Shoa, Central Ethiopia: a cross-sectional, } \\
\text { community-based study }\end{array}$ & $\begin{array}{l}14.2 \%(95 \% \\
\text { Cl: } 11.00- \\
17.00 \%)\end{array}$ & $\begin{array}{l}\text { Home delivery (AOR: } 4.70 ; 95 \% \mathrm{Cl}: 2.56,8.60) \\
\text { Delayed initiation of breastfeeding (AOR: } 5.58 ; 95 \% \\
\text { Cl: } 3.21,9.46 \text { ). } \\
\text { Mothers who can read and write (AOR: } 0.46 ; 95 \% \\
\text { Cl: } 0.22,0.98 \text { ). }\end{array}$ \\
\hline $\begin{array}{l}\text { Asim, } 2020 \\
{[42]}\end{array}$ & Prelacteal feeding practices in Pakistan: a mixed-methods study & $64.7 \%$ & $\begin{array}{l}\text { Birth at public health facilities (AOR: } 0.46 ; 95 \% \text { Cl: } \\
0.02,0.95 \text { ) } \\
\text { Maternal primary education (AOR: } 2.28 ; 95 \% \mathrm{Cl} \text { : } \\
\text { 1.35, 3.85), and } \\
\text { Delayed breastfeeding initiation (AOR: } 0.03 ; 95 \% \\
\text { Cl: } 0.01,0.61 \text { ). }\end{array}$ \\
\hline $\begin{array}{l}\text { Gebremeskel, } \\
2020 \text { [43] }\end{array}$ & $\begin{array}{l}\text { Magnitude of prelacteal feeding and its associated factors among } \\
\text { mothers having children less than one year of age: a community- } \\
\text { based cross-sectional study in rural Eastern Zone, Tigray, Ethiopia }\end{array}$ & $24.7 \%$ & $\begin{array}{l}\text { Parity (AOR }=1.52 ; 95 \% \mathrm{Cl}: 1.04-2.23) \text {, late initiation } \\
\text { of breastfeeding (AOR }=1.83 ; 95 \% \mathrm{Cl}: 1.30-2.59) \text {, } \\
\text { and colostrum discard (AOR }=1.57,95 \% \mathrm{Cl}: 1.06- \\
\text { 2.33). }\end{array}$ \\
\hline $\begin{array}{l}\text { Gao, } 2020 \\
{[44]}\end{array}$ & $\begin{array}{l}\text { Trends in prelacteal feeding practices in rural Bangladesh from } \\
2004 \text { to } 2019\end{array}$ & $88.0 \%$ & $\begin{array}{l}\text { Being having maternal education, improvements } \\
\text { in socioeconomic status, and exposure to media } \\
\text { decreases the odds of prelacteal feeding. }\end{array}$ \\
\hline
\end{tabular}

Note: ANC Antenatal Care, AOR Adjusted Odds Ratio, Cl Confidence Interval

pregnancy, delivery, and postpartum periods to deter prelacteal feeding practices [45-48]. Another possible reason for introducing prelacteal feeding might be since mothers who delivered at home have no the opportunity to access health information about safe breastfeeding practices.

Besides, a woman who delivered by cesarean section was more likely to provide prelacteal feeding. This is in agreement with studies conducted elsewhere [7, 12, 36, $39,40]$. This might be because those mothers may be difficult to give breast milk since they are still recovering from pain, immobilization, and tiredness. This indicates that physicians may not be equipped with the appropriate skills to support mothers under such circumstances.

Mother with a multiple birth was associated with a higher likelihood of prelacteal feeding practice. This is in agreement with a study done in sub-Saharan Africa [15]. This might be because the mother with multiple births perceives their breast milk as insufficient and more likely to practice prelacteal feeding. Regarding the timing of breastfeeding initiation, mothers who had delayed initiation of breastfeeding had higher odds of prelacteal feeding practice compared with their counterparts. This is supported by studies done in Ethiopia, Uganda, and Pakistan [20, 28, 41-43]. This may be justified, as the time between birth and breastfeeding initiation increase, there would be more room for malpractices such as prelacteal feeding.

The study at hand revealed that mothers with a smallsized baby had a higher likelihood of prelacteal feeding practice compared to mothers with large-sized babies. This is in concordance with different studies done elsewhere $[10,12,15,27]$. This may be due to the misconception that small-sized babies will benefit from other foods and liquids.

Mothers who had exposure to different media and mothers from communities with a higher level of media exposure had lower odds of prelacteal feeding practice as compared to their counterparts. This finding is in line with a study done in Nigeria [44]. This may be because disseminating information about the impacts of prelacteal feeding through different media could prevent prelacteal feeding practice. This suggests that printing and electronic mass media play a significant role in fostering optimal breastfeeding practices.

Moreover, women from rural areas had lower odds of prelacteal feeding practice compared to those from urban areas. This is in line with a study done in Egypt [10]. This might be due to the recent expansion of health extension programs among rural people, which 
increases women's level of understanding about the impact of prelacteal feeding on child health.

\section{Strength and limitations of the study}

This study was based on the pooled analysis of the East Africa countries DHS. It was based on a multilevel analytical approach that can able to identify both individual and community-level factors that were associated with prelacteal feeding practice. Also, appropriate estimation adjustments such as weighting were applied. Therefore, the findings of this study will provide important insights to policymakers and governmental and nongovernmental organizations to design the most appropriate interventions at both individual and community levels.

However, this study was not without limitations, in which while interpreting the study findings should be with caution. First, the outcome variable, prelacteal feeding practice was assessed based on the maternal selfreport and therefore there might be a recall bias. Second, DHS did not collect some information such as maternal beliefs, misconceptions, and knowledge towards breastfeeding that were evidenced to influence prelacteal feeding practice. Third, the influence of medicines, including those used for cesarean sections are not assessed. Finally, since it was a cross-sectional study we are unable to assure the temporal relationship between prelacteal feeding practice and important independent variables such as the timing of initiation of breastfeeding.

\section{Conclusion}

In this study, the pooled prevalence of prelacteal feeding is high and still needs strengthening of interventions on appropriate breastfeeding practices. Both individual and community level variables were associated with prelacteal feeding practice. Of individual-level factors, home delivery, multiple birth, cesarean delivery, non-exposure to media, delayed initiation of breastfeeding, and being small-sized baby were associated with higher odds of prelacteal feeding practice. Among community-level factors, rural residence, and higher community-level of media exposure were associated with lower odds of prelacteal feeding practice. Therefore, individual and community-level interventions that encourage mothers to deliver in the health facility and promote timely initiation of breastfeeding are needed to reduce prelacteal feeding practices in east Africa. Moreover, media campaigns regarding this harmful traditional practice are recommended.

\section{Abbreviations}

ANC: Antenatal Care; AOR: Adjusted Odds Ratio; ICC: Intraclass Correlation coefficient; MOR: Median Odds Ratio; PCV: Proportional change in Variance; VIF: Variance Inflation Factor

\section{Acknowledgments}

Our deepest gratitude and appreciation go to the measure DHS program for allowing us to use this survey data.

\section{Authors' contributions}

ABT, MGW, GAT, and ZTT initiated the research concept, analyzed the data, presented and interpreted the results, and wrote up the draft manuscript. All authors read and approved the final manuscript.

\section{Funding}

No funding.

\section{Availability of data and materials}

We included all result-based data within the manuscript and the data set can be accessed online from www.measuredhs.com/data.

\section{Declarations}

\section{Ethics approval and consent to participate}

Since it was based on secondary data, which was publicly available, ethical approval was not required. However, we accessed the data set from the DHS website (https://dhsprogram.com/) through registering or online requesting.

\section{Consent for publication}

Not applicable.

\section{Competing interests}

The authors declare that they have no competing interests.

\section{Author details}

${ }^{1}$ Department of Epidemiology and Biostatistics, Institute of Public Health, College of Medicine and Health Sciences, University of Gondar, Gondar, Ethiopia. ${ }^{2}$ Department of Human Anatomy, University of Gondar, College of Medicine and Health Science, School of Medicine, Gondar, Ethiopia.

Received: 26 August 2020 Accepted: 24 August 2021

Published online: 08 September 2021

\section{References}

1. World Health Organization. Infant and young child feeding: model chapter for textbooks for medical students and allied health professionals: World Health Organization; 2009.

2. Tessema M, Belachew T, Ersino G. Feeding patterns and stunting during early childhood in rural communities of Sidama, South Ethiopia. Pan Afr Med J. 2013;14:75. https://doi.org/10.11604/pamj.2013.14.75.1630.

3. UNICEF. From the first hour of life: making the case for improved infant and young child feeding everywhere. New York: UNICEF; 2016.

4. Pem D. Factors affecting early childhood growth and development: golden 1000 days. J Adv Pract Nurs. 2015;1(101):2573-0347.

5. World Health Organization. Global health risks: mortality and burden of disease attributable to selected major risks: World Health Organization; 2009.

6. Lakati A, Makokha O, Binns C, Kombe Y. The effect of pre-lacteal feeding on full breastfeeding in Nairobi, Kenya. East Afr J Public Health. 2011;7(3):25862.

7. Nguyen PH, Keithly SC, Nguyen NT, Nguyen TT, Tran LM, Hajeebhoy N. Prelacteal feeding practices in Vietnam: challenges and associated factors. BMC Public Health. 2013;13(1):932. https://doi.org/10.1186/1471-2458-13932.

8. Berde AS, Yalcin SS, Ozcebe H, Uner S, Caman OK. Determinants of prelacteal feeding practices in urban and rural Nigeria; a population-based cross-sectional study using the 2013 Nigeria demographic and health survey data. Afr Health Sci. 2017;17(3):690-9. https://doi.org/10.4314/ahs.v1 $7 \mathrm{i} 3.11$.

9. Dalal S, Bansal M, Pande K. Study of infants deaths and pre lacteal feeding practices using verbal autopsy as a tool in deharadun. Natl J Community Med. 2016;7(3):216-8.

10. El-Gilany A-H, Abdel-Hady DM. Newborn first feed and prelacteal feeds in Mansoura, Egypt. BioMed Res Int. 2014;2014:1-7. https://doi.org/10.1155/2 014/258470. 
11. Bansal S, Lalit U, Mahajan R. Prevalence of prelacteal feeding among newborn in rural area. Int J Curr Med Appl Sci. 2016;11(1):30-4.

12. Khanal V, Lee AH, Karkee R, Binns CW. Prevalence and factors associated with prelacteal feeding in Western Nepal. Women Birth. 2016;29(1):12-7. https://doi.org/10.1016/j.wombi.2015.07.006.

13. Legesse M, Demena M, Mesfin F. Haile D. Prelacteal feeding practices and associated factors among mothers of children aged less than 24 months in Raya Kobo district, North Eastern Ethiopia: a cross-sectional study. Int Breastfeed J. 2014;189:1-8.

14. Boccolini CS, Pérez-Escamilla R, Giugliani ERJ, Boccolini P. Inequities in milkbased prelacteal feedings in Latin America and the Caribbean: the role of cesarean section delivery. J Hum Lact. 2015;31(1):89-98. https://doi.org/10.11 77/0890334414559074.

15. Berde AS, Ozcebe H. Risk factors for prelacteal feeding in sub-Saharan Africa: a multilevel analysis of population data from twenty-two countries. Public Health Nutr. 2017;20(11):1953-62. https://doi.org/10.1017/S136898001 7000659.

16. Karkee R, Lee AH, Khanal V, Binns CW. Initiation of breastfeeding and factors associated with prelacteal feeds in Central Nepal. J Hum Lact. 2014;30(3): 353-7. https://doi.org/10.1177/0890334414529845.

17. Teshome B, Kogi-Makau W, Getahun Z, Taye G. Magnitude and determinants of stunting in children underfive years of age in food surplus region of Ethiopia: the case of west gojam zone. Ethiop J Health Dev. 2009; 23(2). https://doi.org/10.4314/ejhd.v23i2.53223.

18. Bekele $Y$, Mengistie B, Mesfine F. Prelacteal feeding practice and associated factors among mothers attending immunization clinic in Harari region public health facilities, eastern Ethiopia. Open J Prev Med. 2014;2014(07): 529-34. https://doi.org/10.4236/ojpm.2014.47063.

19. Hurley WL, Theil PK. Perspectives on immunoglobulins in colostrum and milk. Nutrients. 2011;3(4):442-74. https://doi.org/10.3390/nu3040442.

20. Ogah A, Ajayi A, Akib S, Okolo S. A cross-sectional study of pre-lacteal feeding practice among women attending Kampala International University teaching hospital maternal and child health clinic, Bushenyi, Western Uganda. Asian J Med Sci. 2012;4(3):79-85.

21. Ludvigsson JF. Breastfeeding intentions, patterns, and determinants in infants visiting hospitals in La Paz, Bolivia. BMC Pediatr. 2003;3(1):5. https:// doi.org/10.1186/1471-2431-3-5.

22. Khanal V, Adhikari M, Sauer K, Zhao Y. Factors associated with the introduction of prelacteal feeds in Nepal: findings from the Nepal demographic and health survey 2011. Int Breastfeed J. 2013;8(1):9. https:// doi.org/10.1186/1746-4358-8-9.

23. Raheem RA, Binns CW, Chih HJ, Sauer K. Determinants of the introduction of prelacteal feeds in the Maldives. Breastfeed Med. 2014;9(9):473-8. https:// doi.org/10.1089/bfm.2014.0028.

24. Ibadin O, Ofili N, Monday P, Nwajei C. Prelacteal feeding practices among lactating mothers in Benin City, Nigeria. Nigerian J Paediatr. 2013;40(2):13944. https://doi.org/10.4314/njp.v40i2.6.

25. Engebretsen IMS, Nankabirwa V, Doherty T, Diallo AH, Nankunda J, Fadnes $L T$, et al. Early infant feeding practices in three African countries: the PROMISE-EBF trial promoting exclusive breastfeeding by peer counsellors. Int Breastfeed J. 2014;9(1):19. https://doi.org/10.1186/1746-4358-9-19.

26. Agho KE, Ogeleka P, Ogbo FA, Ezeh OK, Eastwood J, Page A. Trends and predictors of prelacteal feeding practices in Nigeria (2003-2013). Nutrients. 2016;8(8):462. https://doi.org/10.3390/nu8080462.

27. Belachew $A B$, Kahsay $A B$, Abebe YG. Individual and community-level factors associated with introduction of prelacteal feeding in Ethiopia. Arch Public Health. 2016;74(1):6. https://doi.org/10.1186/s13690-016-0117-0.

28. Gualu T, Dilie A, Haile D, Abate A. Determinants of prelacteal feeding practice among postpartum mothers in Debre Markos town, Amhara regional state, Ethiopia, 2016. Nutr Diet Suppl. 2017;9:97-102. https://doi. org/10.2147/NDS.S146188.

29. Croft TN, Marshall AMJ, Allen CK, et al. Guide to DHS Statistics. Rockville: ICF 2018. Availiable from: https://dhsprogram.com/data/Guide-to-DHS-Statistics/ index.cfm

30. The DHS program: DHS Methodology. Available from: http://dhsprogram. com/What-We-Do/Survey-Types/DHS-Methodology.cfm.

31. Central statistical agency (CSA)[Ethiopia] and ICF: Ethiopia demographic and health survey, Addis Ababa, Ethiopia and Calverton, Maryland, USA. 2016.

32. The DHS Program: Sampling and Weighting with DHS Data. 2015. Avialable at https://blog.dhsprogram.com/sampling-weighting-at-dhs/
33. Austin PC, Merlo J. Intermediate and advanced topics in multilevel logistic regression analysis. Stat Med. 2017;36(20):3257-77. https://doi.org/10.1002/ $\operatorname{sim} .7336$.

34. Sommet N, Morselli D. Keep calm and learn multilevel logistic modeling: a simplified three-step procedure using stata, R, Mplus, and SPSS. Int Rev Soc Psychol. 2017;30(1):203-18. https://doi.org/10.5334/irsp.90.

35. Merlo J, Chaix B, Ohlsson H, Beckman A, Johnell K, Hjerpe P, et al. A brief conceptual tutorial of multilevel analysis in social epidemiology: using measures of clustering in multilevel logistic regression to investigate contextual phenomena. J Epidemiol Community Health. 2006;60(4):290-7. https://doi.org/10.1136/jech.2004.029454.

36. Tekaly G, Kassa M, Belete T, Tasew H, Mariye T, Teshale T. Pre-lacteal feeding practice and associated factors among mothers having children less than two years of age in Aksum town, Tigray, Ethiopia, 2017: a cross-sectional study. BMC Pediatr. 2018;18(1):310. https://doi.org/10.1186/s12887-018-12847.

37. Temesgen H, Negesse A, Woyraw W, Getaneh T, Yigizaw M. Prelacteal feeding and associated factors in Ethiopia: systematic review and metaanalysis. Int Breastfeed J. 2018;13(1):49. https://doi.org/10.1186/s13006-0180193-6.

38. Amele EA, Birhanu WD, Desta KW, Woldemariam EB. Prelacteal feeding practice and its associated factors among mothers of children age less than 24 months old in Southern Ethiopia. Ital J Pediatr. 2019;45(1):15. https://doi. org/10.1186/s13052-019-0604-3.

39. Patel A, Banerjee A, Kaletwad A. Factors associated with prelacteal feeding and timely initiation of breastfeeding in hospital-delivered infants in India. J Hum Lact. 2013;29(4):572-8. https://doi.org/10.1177/0890334412474718.

40. Wolde TF, Ayele AD, Takele WW. Prelacteal feeding and associated factors among mothers having children less than 24 months of age, in Mettu district, Southwest Ethiopia: a community based cross-sectional study. BMC Res Notes. 2019;12(1):9. https://doi.org/10.1186/s13104-019-4044-3.

41. Argaw MD, Asfaw MM, Ayalew MB, Desta BF, Mavundla TR, Gidebo KD, et al Factors associated with prelacteal feeding practices in Debre Berhan district, north Shoa, Central Ethiopia: a cross-sectional, community-based study. BMC Nutr. 2019;5(1):14. https://doi.org/10.1186/s40795-019-0277-8.

42. Asim M, Ahmed ZH, Hayward MD, Widen EM. Prelacteal feeding practices in Pakistan: a mixed-methods study. Int Breastfeed J. 2020;15(1):53. https://doi. org/10.1186/s13006-020-00295-8.

43. Gebremeskel SG, Gebru TT, Kassahun SS, Gebrehiwot BG. Magnitude of prelacteal feeding and its associated factors among mothers having children less than one year of age: a community-based cross-sectional study in rural eastern zone, Tigray, Ethiopia. Adv Public Health. 2020;2020:17. https://doi.org/10.1155/2020/4926890.

44. Gao Y, Palmer A, Thorne-Lyman A, Shaikh S, Ali H, Tong H, et al. Trends in prelacteal feeding practices in rural Bangladesh from 2004-2019. Curr Dev Nutr. 2020;4(2). https://doi.org/10.1093/cdn/nzaa053_034.

45. Lind JN, Ahluwalia IB, Perrine CG, Li R, Harrison L, Grummer-Strawn LM. Prenatal breastfeeding counseling--pregnancy risk assessment monitoring system, United States, 2010. MMWR Suppl. 2014;63(2):14-9.

46. World Health Organization: Counselling for maternal and newborn health care: A handbook for building skills. World Health Organization; 2010.

47. Edwards RA, Colchamiro R, Tolan E, Browne S, Foley M, Jenkins L, et al. Online continuing education for expanding clinicians' roles in breastfeeding support. J Hum Lact. 2015;31(4):582-6. https://doi.org/10.1177/089033441 5585977.

48. World Health Organization. Guideline: counselling of women to improve breastfeeding practices: World Health Organization; 2018.

\section{Publisher's Note}

Springer Nature remains neutral with regard to jurisdictional claims in published maps and institutional affiliations. 